\title{
A Hybrid MLFMM-UTD Method for the Solution of Very Large 2-D Electromagnetic Problems
}

\author{
Giorgos Karagounis, Daniël De Zutter, Fellow, IEEE, and Dries Vande Ginste, Senior Member, IEEE
}

\begin{abstract}
The multilevel fast multipole method (MLFMM) is combined with the uniform theory of diffraction (UTD) to model two-dimensional (2-D) scattering problems including very large scatterers. The discretization of the very large scatterers is avoided by using ray-based methods. Reflections are accounted for by image source theory, while for diffraction a new MLFMM translation matrix is introduced. The translation matrix elements are derived based on a technique that generalizes the use of UTD for arbitrary source configurations and that efficiently describes the field over extended regions of space. $\mathcal{O}(n)$ scaling of the computational time and memory requirements is achieved for relevant structures, such as large antenna arrays in the presence of a wedge. The theory is validated by means of several illustrative numerical examples and is shown to remain accurate for non-line-of-sight (NLoS) scattering problems.
\end{abstract}

Index Terms-Boundary integral methods, geometrical optics (GO), hybrid solution methods, method of moments (MoM), multilevel fast multipole method (MLFMM), uniform theory of diffraction (UTD).

\section{INTRODUCTION}

$\mathbf{E}$ LECTROMAGNETIC (EM) solvers provide a means to deal with scattering problems in complex environments. For scattering problems involving piecewise homogeneous domains, boundary integral equations (BIEs) are advantageous as only the unknown tangential fields on the boundary surfaces between domains have to be discretized. Discretizing the BIEs according to a method of moments (MoM) scheme results in a system with $N$ unknowns and $N$ equations that can be solved efficiently using an iterative solver. The memory requirements and the computational complexity of the matrix-vector multiplication scale as $\mathcal{O}\left(N^{2}\right)$. Multilevel fast multipole methods (MLFMMs) reduce this scaling to $\mathcal{O}(N)$ or $\mathcal{O}(N \log N)$ [1], depending on the specific geometry of the problem. When the problem size becomes very large, memory requirements will, however, still grow excessively. High-frequency methods are useful in this case, as they provide asymptotic solutions to account for the presence of very large scatterers. Hybrid methods that combine MoM and high-frequency approaches are able

Manuscript received October 01, 2014; revised September 22, 2015; accepted November 15, 2015. Date of publication November 19, 2015; date of current version December 31, 2015. This work was supported by the Research Foundation Flanders (FWO).

The authors are with the IBCN/Electromagnetics Group, Department of Information Technology, Ghent University/iMinds, 9000 B-Gent, Belgium (e-mail: giorgos.karagounis@UGent.be).

Color versions of one or more of the figures in this paper are available online at http://ieeexplore.iee.org.

Digital Object Identifier 10.1109/TAP.2015.2501812 to accurately deal with very large EM problems in the presence of complex geometries.

Field-based hybrid methods that combine the geometrical theory of diffraction (GTD) or the uniform theory of diffraction (UTD) with MoM can avoid the discretization of large scatterers completely [2]-[8]. The current-based physical theory of diffraction (PTD) is combined with MoM in [9]-[13]. The number of unknowns is reduced greatly on large smooth scatterers by only introducing MoM basis functions at discontinuities (points of diffraction). The matrix-vector multiplication time and the memory requirements of these methods are $\mathcal{O}\left(n^{2}\right)$, where $n$ is now the number of unknowns obtained by only discretizing the boundaries of the scatterers treated by the BIE-MoM part of the hybrid scheme.

A hybrid MLFMM-UTD method has been presented in [14]. The method estimates the amplitude and phase of the UTD rays based on the MLFMM radiation pattern. This approximation proves sufficient to compute the amplitude of the total field in regions illuminated by the source. Here, we present an MLFMM-UTD hybrid method that provides accurate solutions in all regions where UTD is applicable. The novel method is based on a technique that generalizes the use of UTD for general source configurations and efficiently describes the field over extended regions of space [15]. Reflections at straight PEC scatterers are taken exactly into account using image source theory. For compact scatterers, such as large antenna arrays, the memory requirements, and the computational time scale as $\mathcal{O}(n)$. Therefore, our method provides a valid and efficient alternative if the phase of the field also needs to be described accurately and for nonline-of-sight (NLoS) problems.

In Section II, we present the new MLFMM-UTD method, preceded by a short review of the classical MLFMM. Also, a theoretical derivation of the proposed technique's computational complexity is given. Two numerical examples in Section III prove the accuracy of the method and demonstrate the good scaling properties of the algorithm. A third example illustrates the versatility of the method. Conclusion is formulated in Section IV.

In this paper, we deal with two-dimensional (2-D) transverse magnetic (TM) problems. The $z$-axis is chosen as the axis of invariance. $A n \exp (j \omega t)$ time dependence, with $\omega$ being the angular frequency, is assumed and suppressed throughout the text. Unit vectors are denoted by a hat, e.g., $\hat{\boldsymbol{x}}$.

\section{FORMALISM}

We consider an environment with $N_{S}$ PEC scatterers. Their boundaries are denoted by $c_{i}\left(i=1, \ldots, N_{S}\right)$. The electric field 
integral equation (EFIE) is then given by

$$
E_{z}^{i n c}(\boldsymbol{\rho})=-j \omega \mu_{0} \sum_{i=1}^{N_{S}} \oint_{c_{i}} \mathrm{~d} \boldsymbol{\rho}^{\prime} G\left(\boldsymbol{\rho} ; \boldsymbol{\rho}^{\prime}\right) J_{z}\left(\boldsymbol{\rho}^{\prime}\right)
$$

where $\mu_{0}$ is the magnetic permeability of the (nonmagnetic) background medium, $J_{z}\left(\boldsymbol{\rho}^{\prime}\right)$ is the unknown current density on the surface of the scatterers, $G\left(\boldsymbol{\rho} ; \boldsymbol{\rho}^{\prime}\right)$ is the Green's function for a line source residing at $\rho^{\prime}, \rho$ is a point on the surface of the scatterers, and $E_{z}^{i n c}(\boldsymbol{\rho})$ is the incident field due to an excitation source. In a classical MoM scheme, as the background medium does not contain any scatterers, the Green's function used in (1) is the free space Green's function $G^{\text {free }}\left(\boldsymbol{\rho} ; \boldsymbol{\rho}^{\prime}\right)$, given by

$$
G^{f r e e}\left(\boldsymbol{\rho} ; \boldsymbol{\rho}^{\prime}\right)=\frac{j}{4} H_{0}^{(2)}\left(k\left\|\boldsymbol{\rho}-\boldsymbol{\rho}^{\prime}\right\|\right)
$$

where $k$ is the wavenumber of the background medium and $\|\cdot\|$ denotes the vector norm.

In a hybrid MoM-UTD scheme, some of the scatterers for which a canonical UTD solution is available are not discretized. This is shown in Fig. 1. Their presence is taken into account by adjusting the Green's function for ray-optical contributions, such as reflections and diffractions. The Green's function to be used in (1) then becomes

$$
\begin{aligned}
G^{U T D}\left(\boldsymbol{\rho} ; \boldsymbol{\rho}^{\prime}\right)= & G^{\text {free }}\left(\boldsymbol{\rho} ; \boldsymbol{\rho}^{\prime}\right)+G^{\text {refl }}\left(\boldsymbol{\rho} ; \boldsymbol{\rho}^{\prime}\right) \\
& +G^{\text {diff }}\left(\boldsymbol{\rho} ; \boldsymbol{\rho}^{\prime}\right)+\cdots
\end{aligned}
$$

Higher order interactions, such as reflection-reflection, diffraction-reflection, etc., can be further added if necessary.

After discretization of the boundaries of all remaining scatterers, a linear system with $n$ equations and $n$ unknowns of the following form is derived from (1):

$$
\boldsymbol{V}=\boldsymbol{Z}^{\text {tot }} \cdot \boldsymbol{I}=\boldsymbol{Z}^{\text {free }} \cdot \boldsymbol{I}+\boldsymbol{Z}^{\text {refl }} \cdot \boldsymbol{I}+\boldsymbol{Z}^{\text {diff }} \cdot \boldsymbol{I}+\cdots
$$

This system is solved for the unknown current vector $\boldsymbol{I}$. Iterative solvers seek at each iteration a better approximation for this vector, based on the matrix-vector product of $Z^{\text {tot }}$ with the previous approximation. An efficient matrix-vector multiplication scheme for each term in the right-hand side (RHS) of (4) is presented in what follows.

\section{A. Direct Coupling}

The contributions due to direct coupling, i.e., the first term in the RHS of (4), can be handled by conventional MLFMM. The reader is encouraged to consult [1] and the references therein to gain familiarity with the MLFMM scheme. In this section, we will only repeat the gist of it, in order to introduce some notation and terminology that is necessary for the comprehension of what follows.

First, the boundaries of all relevant scatterers are discretized into $n$ finite segments, corresponding to the pertinent BIEMoM approach. Next, all these segments are enclosed in boxes in a so-called $L$-level MLFMM quad-tree. At each level

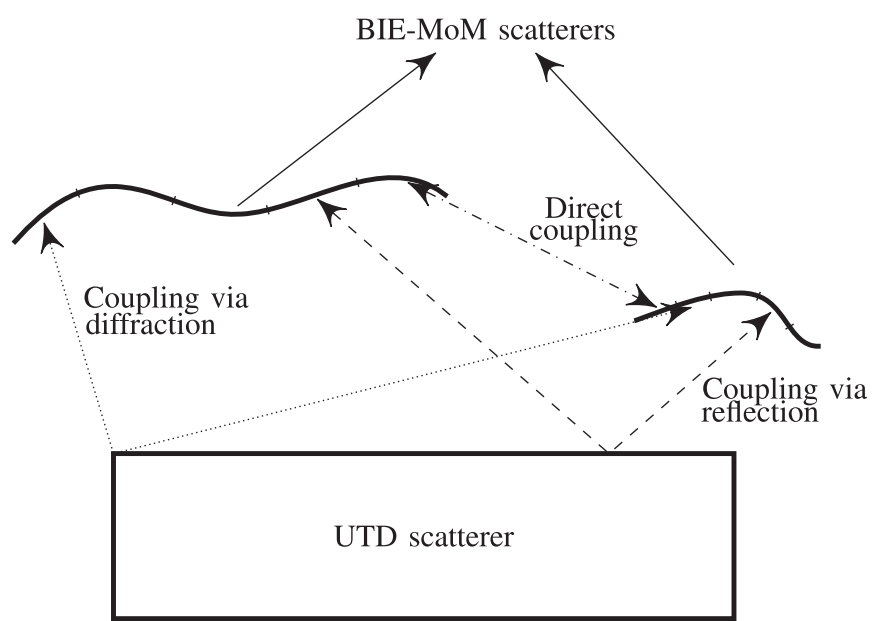

Fig. 1. Generic example of the MoM-UTD method. The BIE-MoM scatterers are discretized, while the presence of the UTD scatterer is taken into account implicitly.

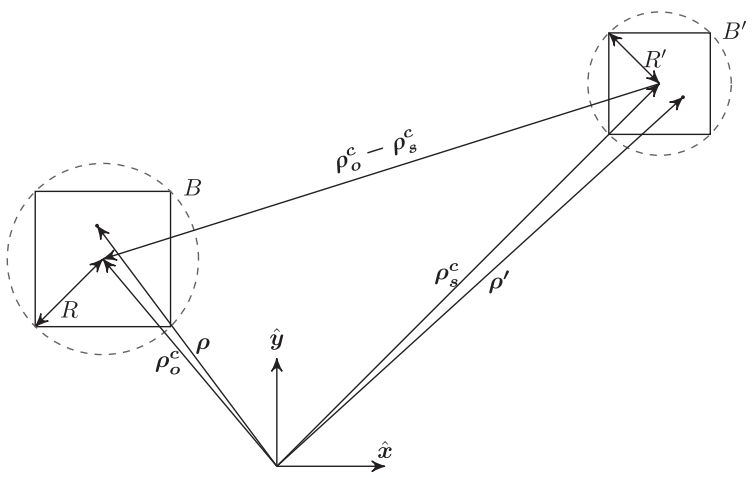

Fig. 2. Illustration of the contribution due to direct coupling: typical MLFMM constellation of a source box $B^{\prime}$ and an observation box $B$.

$l=1,2, \ldots, L$, the boxes are circumscribed by a hypothetical circle of radius $R_{l}$.

Any interaction between two segments in the BIE-MoM scheme is readily rewritten as the interaction between many elementary line sources $s_{i}$ with strength $J_{s_{i}}$. In Fig. 2, one such single line source, located at $\rho^{\prime}$ and residing in source box $B^{\prime}$, and a single observer, located at $\rho$ and residing in observation box $B$, is shown. The centers of the source and observation box are located at $\boldsymbol{\rho}_{s}^{c}$ and $\boldsymbol{\rho}_{o}^{c}$, respectively.

First, during the so-called aggregation step, the radiation pattern of box $B^{\prime}$ is sampled into $2 Q+1$ outgoing plane waves (OPWs) as follows:

$$
\mathrm{OPW}_{q^{\prime}}^{B^{\prime}}=\sum_{s_{i}} e^{j \boldsymbol{k}\left(\phi_{q^{\prime}}\right) \cdot\left(\boldsymbol{\rho}^{\prime}-\boldsymbol{\rho}_{s}^{c}\right)} J_{s_{i}}, q^{\prime}=-Q, \ldots, Q
$$

where $\boldsymbol{k}\left(\phi_{q^{\prime}}\right)=k\left(\cos \phi_{q^{\prime}} \hat{\boldsymbol{x}}+\sin \phi_{q^{\prime}} \hat{\boldsymbol{y}}\right)$. The samples are taken at angles $\phi_{q^{\prime}}=2 \pi q^{\prime} /(2 Q+1), q^{\prime}=-Q, \ldots, Q$, and the number of samples is typically chosen such that the radiation pattern can be reconstructed with a desired number of digits of accuracy, denoted by $d_{0}$. In a well-constructed MLFMM tree, any accuracy up to machine precision can be reached [1], provided $Q$ is chosen to be

$$
Q=2 k R^{\prime}+1.8 d_{0}^{2 / 3}\left(2 k R^{\prime}\right)^{1 / 3} .
$$


Second, a translation matrix T converts the OPWs about the center of box $B^{\prime}$ to incoming plane waves (IPWs) about the center of box $B$ as follows:

$$
\mathrm{IPW}_{q}^{B}=\sum_{q^{\prime}=-Q}^{Q} T_{q q^{\prime}} \mathrm{OPW}_{q^{\prime}}^{B^{\prime}}
$$

where the numbers $T_{q q^{\prime}}$ represent the elements of the translation matrix. In the case of direct coupling, treated with MLFMM, this matrix $\mathrm{T}$ is diagonal and its $2 Q+1$ nonzero elements are given by

$$
T_{q q}(k, r, \phi)=\frac{1}{2 Q+1} \sum_{q^{\prime \prime}=-Q}^{Q} H_{q^{\prime \prime}}^{(2)}(k r) e^{j q^{\prime \prime}\left(\phi-\phi_{q}-\frac{\pi}{2}\right)}
$$

where $q=-Q, \ldots, Q$ and with $r=\left\|\boldsymbol{\rho}_{o}^{c}-\boldsymbol{\rho}_{s}^{c}\right\|$ the distance between the centers of the boxes and $\phi$ the angle that vector $\boldsymbol{\rho}_{o}^{c}-\boldsymbol{\rho}_{s}^{c}$ makes with the $x$-axis.

Third, during the disaggregation step, the IPWs are evaluated at the observation points. In case of a single, elementary line source at $\boldsymbol{\rho}^{\prime}$, the field at a single observer at $\rho$ in box $B$ is nothing else than the pertinent Green's function, now expanded using the plane wave formalism as follows:

$$
G^{\text {free }}\left(\boldsymbol{\rho} ; \boldsymbol{\rho}^{\prime}\right)=\frac{j}{4} \sum_{q=-Q}^{Q} e^{-j \boldsymbol{k}\left(\phi_{q}\right) \cdot\left(\boldsymbol{\rho}-\boldsymbol{\rho}_{o}^{c}\right)} \mathrm{IPW}_{q}^{B} .
$$

To obtain an efficient multilevel scheme, the interaction between boxes happens at well-chosen levels in the MLFMM tree. Upsampling and downsampling of OPWs and IPWs happen, e.g., via fast Fourier transforms (FFTs).

The above scheme is only used for the direct coupling contribution when box $B$ lies in line-of-sight $(\operatorname{LoS})$ of box $B^{\prime}$.

\section{B. Coupling by Reflection}

The second term in the RHS of (4) is taken into account using image source theory. An illustration of a reflection at a UTD object is given in Fig. 3. Adopting an MLFMM implementation again, the radiation pattern of the image box $B^{\prime \prime}$ is to be translated to box $B$. The translation matrix is the same as in (8), where the distance $r$ and angle $\phi$ are now measured from the center of box $B^{\prime \prime}$. An interesting property is that the radiation pattern of the image box $B^{\prime \prime}$ can be expressed in terms of the radiation pattern of $B^{\prime}$. Indeed, from (5) and using some basic vector algebra, for the reflection at a straight PEC scatterer elevated at angle $\psi$, the following holds:

$$
\mathrm{OPW}_{q^{\prime}}^{B^{\prime \prime}}=-\mathcal{R}_{2 \psi} \mathrm{OPW}_{-q^{\prime}}^{B^{\prime}}
$$

where $\mathcal{R}_{2 \psi}$ denotes the rotation operation over an angle $2 \psi$. Such rotations are accurately and efficiently computed by using the FFT scheme shown in Fig. 4 [16]. The computational complexity remains unaffected by this additional step.

\section{Coupling by Diffraction}

The third term in the RHS of (4) deserves some more attention. Since canonical UTD solutions only exist for a limited

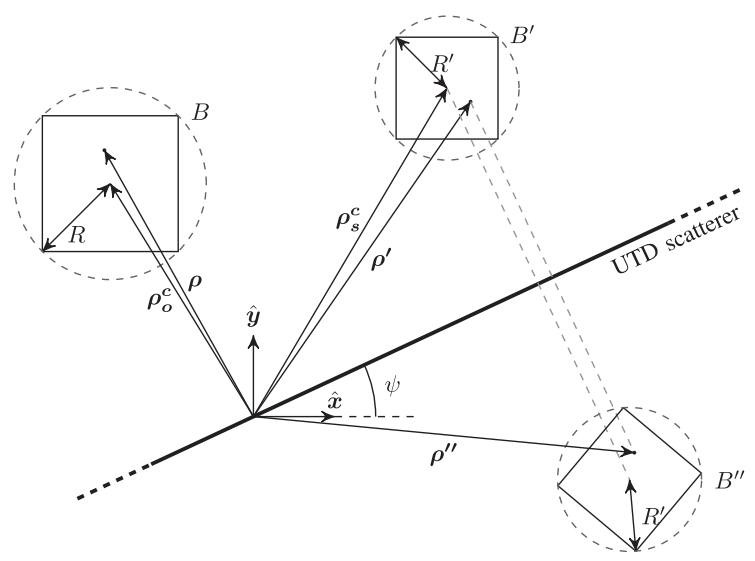

Fig. 3. Illustration of the reflection at a long straight surface of a UTD scatterer. The surface makes an angle $\psi$ with the $x$-axis. The image of a source $\boldsymbol{\rho}^{\prime}$ in source box $B^{\prime}$ is then $\boldsymbol{\rho}^{\prime \prime}$ in image box $B^{\prime \prime}$. Reflections are now described by the interaction between boxes $B$ and $B^{\prime \prime}$.

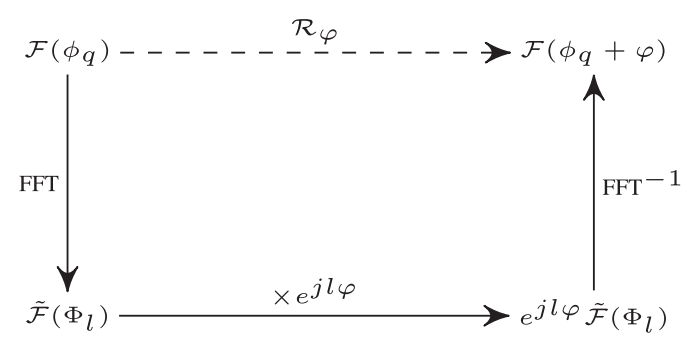

Fig. 4. Efficient rotation of a sampled function $\mathcal{F}$ over an angle $\varphi$ by the use of FFTs.

number of illumination sources, such as a line source, it is not possible to use UTD directly in an MLFMM setting. Therefore, in the envisaged hybrid scheme, we propose to generalize the UTD method such that: 1) we can deal with arbitrary sources, in particular MLFMM source boxes and 2) the diffracted field can be translated to IPWs at observation boxes. The UTD method described in [15] serves as a starting point. The physics of the method is summarized as follows.

1) The arbitrary source is replaced by an equivalent set of Huyghens' line sources residing on the source's circumscribing circle.

2) The source field is expanded into multipoles and is related to the expansion in Huyghens' sources.

3) For each of these Huyghens' line sources, UTD diffraction is applied.

4) The diffracted field is expanded into multipoles inside an arbitrary observation region.

5) A set of coupling coefficients is introduced that relates the multipole expansion of the source field to that of the diffracted field.

Now, we adopt and extend this scheme to make it suitable for integration within an MLFMM solver. Consider the configuration in Fig. 5. A source box $B^{\prime}$, circumscribed by a circle $\mathcal{C}^{\prime}$ with radius $R^{\prime}$, resides at position $\rho_{s}^{c}$ with respect to the diffracting tip of a PEC wedge with interior angle $\alpha$. A similar notation is used for the observation box $B$. The incident field due to the arbitrary source configuration inside box $B^{\prime}$ is 
expanded into multipoles as follows:

$$
E^{i n c}\left(\boldsymbol{\rho}_{\boldsymbol{o}}\right)=-\frac{\omega \mu_{0}}{4} \sum_{q^{\prime}=-Q^{\prime}}^{Q^{\prime}} a_{q^{\prime}} H_{q^{\prime}}^{(2)}\left(k\left\|\boldsymbol{\rho}_{\boldsymbol{o}}-\boldsymbol{\rho}_{\boldsymbol{s}}^{\boldsymbol{c}}\right\|\right) e^{j q^{\prime} \phi_{o}}
$$

for every observation point at position $\rho_{o}$ outside $\mathcal{C}^{\prime}\left(\| \rho_{o}-\right.$ $\left.\rho_{s}^{c} \|>R^{\prime}\right) . \phi_{o}$ is the angle that $\rho_{o}-\rho_{s}^{c}$ makes with respect to the $x$-axis. All coefficients $a_{q^{\prime}}$ are independent of $\rho_{o}$. The number of multipoles $2 Q^{\prime}+1$ again depends on the size $R^{\prime}$ of box $B^{\prime}$.

The diffracted field inside the observation box $B$ is also expanded into multipoles, i.e.,

$$
E^{\operatorname{diff}}(\boldsymbol{\rho})=-\frac{\omega \mu_{0}}{4} \sum_{q=-Q}^{Q} b_{q} J_{q}\left(k\left\|\boldsymbol{\rho}-\boldsymbol{\rho}_{\boldsymbol{o}}^{\boldsymbol{c}}\right\|\right) e^{j q \phi} .
$$

The angle $\phi$ is the angle that $\boldsymbol{\rho}-\boldsymbol{\rho}_{\mathrm{o}}^{c}$ makes with the $x$-axis. The number of multipoles $2 Q+1$ depends on $R$ and, for generality, we describe the case where $R \neq R^{\prime}$ and thus $Q \neq Q^{\prime}$ (e.g., when $B$ and $B^{\prime}$ belong to different MLFMM levels). Via the method described in [15], the two multipole expansions are shown to be related through

$$
b_{q}=\sum_{q^{\prime}=Q^{\prime}}^{Q^{\prime}} t_{q q^{\prime}} a_{q^{\prime}}
$$

The coupling coefficients $t_{q q^{\prime}}$ is given by

$$
\begin{aligned}
t_{q q^{\prime}}= & \frac{1}{J_{q}(k R)} \frac{1}{J_{q^{\prime}}\left(k R^{\prime}\right)} \sum_{n=-Q}^{Q} H_{n}^{(2)}\left(k \rho_{o}^{c}\right) J_{n}(k R) e^{-j n \phi_{o}^{c}} \\
& \times \sum_{m=-Q^{\prime}}^{Q^{\prime}} \frac{1-j}{2} \sqrt{\pi k} d_{-n+q, m-q^{\prime}} \\
& \times H_{m}^{(2)}\left(k \rho_{s}^{c}\right) J_{m}\left(k R^{\prime}\right) e^{j m \phi_{s}^{c}}
\end{aligned}
$$

where $q^{\prime}=-Q^{\prime}, \ldots, Q^{\prime}$ and $q=-Q, \ldots, Q$. The angle $\phi_{s}^{c}$ is the angle that $-\rho_{s}^{c}$ makes with the $x$-axis. The coefficients $d_{-n+q, m-q^{\prime}}$ are given by

$$
d_{s, l}=\frac{1}{4 \pi^{2}} \int_{\mathcal{C}} \mathrm{d} \phi_{b} e^{-j s \phi_{b}} \int_{\mathcal{C}^{\prime}} \mathrm{d} \phi_{b}^{\prime} e^{-j l \phi_{b}^{\prime}} D_{U T D}\left(L ; \boldsymbol{R}^{\prime}, \boldsymbol{R}\right) .
$$

The vector $\boldsymbol{R}$ denotes a position on $\mathcal{C}$, measured from the center $\rho_{o}^{c}$ and at angle $\phi_{b}$. The parameter $L$ in (15) is the wellknown length parameter for line source illumination

$$
L=\frac{\left\|\rho_{s}^{c}+R^{\prime}\right\| \cdot\left\|\rho_{o}^{c}+R\right\|}{\left\|\rho_{s}^{c}+R^{\prime}\right\|+\left\|\rho_{o}^{c}+R\right\|} .
$$

Now the coefficients $a_{q^{\prime}}$ of the expansion (11) are written in terms of OPWs and Bessel's integral is invoked to deal with $J_{q}\left(k\left\|\rho-\rho_{o}^{c}\right\|\right)$ in (12). The mathematical details are given in Appendixes A and B. By introduction of the results (A9) and (B2) into (12), the diffracted field is written in a form that is compatible with MLFMM, i.e.

$$
E^{\operatorname{diff}}(\boldsymbol{\rho})=-\frac{\omega \mu_{0}}{4} \sum_{p=-Q}^{Q} \operatorname{IPW}_{p} e^{-j \boldsymbol{k}\left(\phi_{p}\right) \cdot\left(\boldsymbol{\rho}-\boldsymbol{\rho}_{o}^{c}\right)}
$$

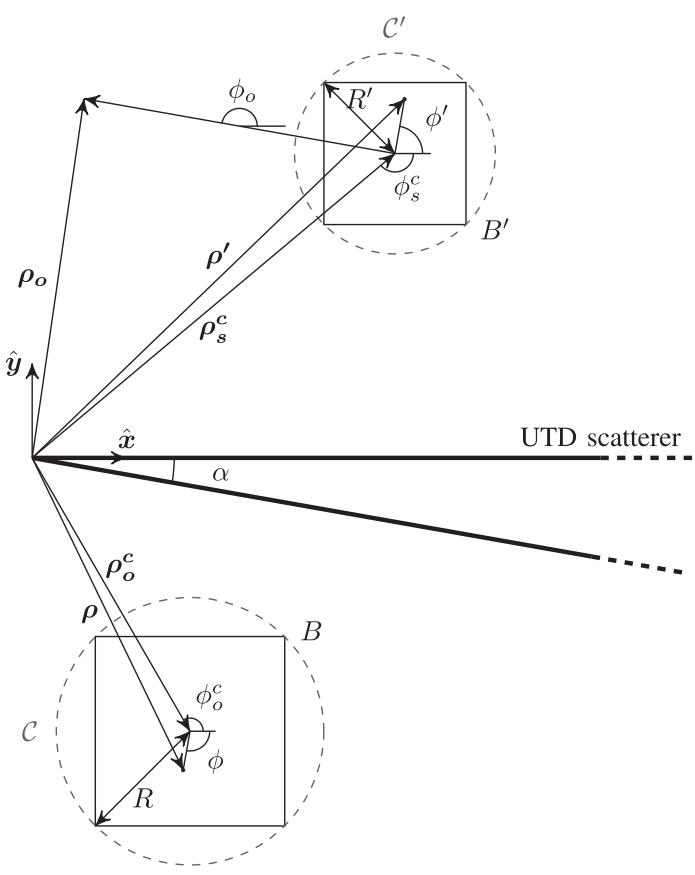

Fig. 5. Illustration of the MLFMM-UTD geometry, where the UTD object is a PEC wedge with opening angle $\alpha$ situated between the MLFMM boxes.

where the IPWs are now described by

$$
\mathrm{IPW}_{p}=\sum_{p^{\prime}=-Q^{\prime}}^{Q^{\prime}} \tilde{T}_{p p^{\prime}} \mathrm{OPW}_{p^{\prime}}, p=-Q, \ldots, Q
$$

and the elements $\tilde{T}_{p p^{\prime}}$ of the new translator $\tilde{\mathrm{T}}$ are given by

$$
\begin{aligned}
\tilde{T}_{p p^{\prime}}= & \frac{1}{(2 Q+1)\left(2 Q^{\prime}+1\right)} \\
& \times \sum_{q=-Q}^{Q} \sum_{q^{\prime}=-Q^{\prime}}^{Q^{\prime}} e^{j q\left(\phi_{p}+\pi / 2\right)} t_{q q^{\prime}} e^{-j q^{\prime}\left(\phi_{p^{\prime}}+\pi / 2\right)}
\end{aligned}
$$

where $p^{\prime}=-Q^{\prime}, \ldots, Q^{\prime}$ and $p=-Q, \ldots, Q$. Note that, in contrast to the conventional MLFMM translator T in (8) used to describe the direct coupling (Section II-A), this new translator $\tilde{\mathrm{T}}$ is not diagonal, nor sparse. Nevertheless, in the next sections, it will be shown that it can be computed efficiently during the setup of the algorithm and that the desired low computational complexity can be maintained.

\section{Efficient Computation of the New Translator $\tilde{\mathbf{T}}$}

The computation of the coupling coefficients $t_{q q^{\prime}}$ requires a double summation for every $q$ and $q^{\prime}$. Nonetheless, as the matrix with elements $d_{-n+q, m-q^{\prime}}$ in (14) is Toeplitz, the matrix of coupling coefficients $t_{q q^{\prime}}$ can still be computed in an efficient way following a routine similar to the one presented in [17]. Consider the following identities:

$$
1 \equiv \sum_{s=-2 Q}^{2 Q} \delta_{s, q-n}, \forall q, n \in[-Q, Q]
$$




$$
\delta_{s, q-n} \equiv \frac{1}{4 Q+1} \sum_{t=-2 Q}^{2 Q} e^{-j \frac{2 \pi t}{4 Q+1}[s-(q-n)]} .
$$

By using both identities, it is readily shown that (14) can be written as

$$
\begin{aligned}
t_{q q^{\prime}} & =\frac{1}{J_{q}(k R) J_{q^{\prime}}\left(k R^{\prime}\right)} \frac{1}{(4 Q+1)\left(4 Q^{\prime}+1\right)} \\
& \times \sum_{u=-2 Q}^{2 Q} \sum_{v=-2 Q^{\prime}}^{2 Q^{\prime}}\left[\sum_{n=-Q}^{Q} H_{n}^{(2)}\left(k \rho_{o}^{c}\right) J_{n}(k R) e^{-j n\left(\phi_{o}^{c}-\phi_{u}\right)}\right] \\
& \times \frac{1-j}{2} \sqrt{\pi k}\left[\sum_{s=-2 Q}^{2 Q} \sum_{l=-2 Q^{\prime}}^{2 Q^{\prime}} d_{s, l} e^{j s \phi_{u}} e^{j l \phi_{v}}\right] \\
& \times\left[\sum_{m=-Q^{\prime}}^{Q^{\prime}} H_{m}^{(2)}\left(k \rho_{s}^{c}\right) J_{m}\left(k R^{\prime}\right) e^{j m\left(\phi_{s}^{c}-\phi_{v}\right)}\right] e^{-j q \phi_{u}} e^{j q^{\prime} \phi_{v}}
\end{aligned}
$$

where $\phi_{u}=2 \pi u /(4 Q+1)$ and $\phi_{v}=2 \pi v /\left(4 Q^{\prime}+1\right)$. The expression between square brackets in the third line inverts the FFT that appears in definition (15) of the coefficients $d_{s, l}$. In the second and fourth lines, one recognizes Graf's addition theorem [18]. Expression (22) thus reduces to

$$
\begin{aligned}
t_{q q^{\prime}}= & \frac{1}{J_{q}(k R) J_{q^{\prime}}\left(k R^{\prime}\right)} \frac{1}{(4 Q+1)\left(4 Q^{\prime}+1\right)} \\
& \times \sum_{u=-2 Q}^{2 Q} \sum_{v=-2 Q^{\prime}}^{2 Q^{\prime}} e^{-j q \phi_{u}} e^{j q^{\prime} \phi_{v}} \\
& \times \frac{1-j}{2} \sqrt{\pi k} H_{0}^{(2)}\left(k\left\|\rho_{\boldsymbol{o}}^{c}+\boldsymbol{R}\left(\phi_{u}\right)\right\|\right) \\
& \times D_{U T D}\left(L ; \boldsymbol{R}^{\prime}\left(\phi_{v}\right), \boldsymbol{R}\left(\phi_{u}\right)\right) \\
& \times H_{0}^{(2)}\left(k\left\|\boldsymbol{\rho}_{\boldsymbol{s}}^{\boldsymbol{c}}+\boldsymbol{R}^{\prime}\left(\phi_{v}\right)\right\|\right)
\end{aligned}
$$

where $\boldsymbol{R}^{\prime}\left(\phi_{v}\right)$ is a vector with length $R^{\prime}$ that makes an angle $\phi_{v}$ with the positive $x$-axis; similarly for $\boldsymbol{R}\left(\phi_{u}\right)$. The factor in the third line of (23) originates from an approximation that was introduced in [15] and is identical to (A1) with $\nu=0$. This approximation can now be undone. We further substitute $v$ by $-v$. The final expression for the coefficients $t_{q q^{\prime}}$ becomes

$$
\begin{aligned}
& t_{q q^{\prime}}=\frac{1}{J_{q}(k R) J_{q^{\prime}}\left(k R^{\prime}\right)} \frac{1}{(4 Q+1)\left(4 Q^{\prime}+1\right)} \\
& \times \sum_{u=-2 Q}^{2 Q} \sum_{v=-2 Q^{\prime}}^{2 Q^{\prime}} e^{-j q \phi_{u}} e^{-j q^{\prime} \phi_{v}} \frac{e^{-j k\left\|\boldsymbol{\rho}_{o}^{c}+\boldsymbol{R}\left(\phi_{u}\right)\right\|}}{\sqrt{\left\|\boldsymbol{\rho}_{\boldsymbol{o}}^{\boldsymbol{c}}+\boldsymbol{R}\left(\phi_{u}\right)\right\|}} \\
& \times D_{U T D}\left(L ; \boldsymbol{R}^{\prime}\left(-\phi_{v}\right), \boldsymbol{R}\left(\phi_{u}\right)\right) H_{0}^{(2)}\left(k\left\|\boldsymbol{\rho}_{\boldsymbol{s}}^{\boldsymbol{c}}+\boldsymbol{R}^{\prime}\left(-\phi_{v}\right)\right\|\right) .
\end{aligned}
$$

Thus, these coefficients can now be efficiently computed by means of a 2-D FFT. Once they are known, $\tilde{T}$ in (19) can also be efficiently computed by a 2-D FFT.

\section{E. Computational Complexity}

The interactions due to direct coupling are taken into account by using a traditional MLFMM scheme. The complexity depends on the geometry of the scatterers. If the scatterers are

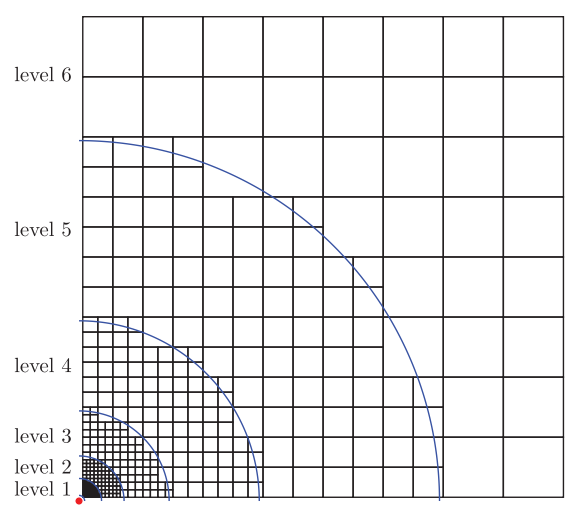

Fig. 6. Boxes of a tree that interacts with one another through diffraction by means of MLFMM. The diffraction tip is shown in red. The blue arcs show the admission boundaries for different levels in the MLFMM tree.

densely packed volume scatterers, such that the boxes of the quad-tree are fully filled, the complexity of traditional MLFMM methods is $\mathcal{O}(n)$ [1].

The coupling due to reflections follows the scheme of the traditional MLFMM, except for the additional conversion of the radiation pattern of a source box to that of the corresponding image source box. This operation is similar to the upsampling and downsampling of the radiation pattern by FFTs in MLFMM. Hence, this additional step does not alter the $\mathcal{O}(n)$ complexity.

The essential difference between the presented MLFMMUTD method and the traditional MLFMM scheme manifests itself during the translation step of the diffraction coupling. Our algorithm uses an adaptive scheme that allows higher level boxes, that are further away from the diffracting object, to interact with lower level boxes, that reside closer to it. A box is considered to be far from the tip when the distance between the center of the box and the diffraction tip is about five times the box size and when this is not the case for its parent box. An example is shown in Fig. 6. The circular arcs in the figure show the boundaries beyond which the diffraction coupling can be treated at a higher MLFMM level.

The computational cost of the new scheme is now calculated. Because the area between two arcs scales linearly with the size of the boxes it encompasses, the number of boxes at each level is bounded. We denote this as upper limit $C_{\text {int }}$. Moreover, a translation between two boxes at levels $l$ and $l^{\prime}$ costs $\left(2 Q_{l}+1\right)\left(2 Q_{l^{\prime}}+1\right)$ operations, where $2 Q_{l}+1$ is the sampling rate at the corresponding level $l$. Due to (6), this sampling rate scales as $\mathcal{O}(R)$, with $R$ the radius of the box. For densely packed volume scatterers, the number of unknowns $n_{b}$ in a box is proportional to the area of that box. Therefore, $R \propto$ $\sqrt{n_{b}}$ and $\left(2 Q_{l}+1\right)\left(2 Q_{l^{\prime}}+1\right) \propto \sqrt{n_{l} n_{l^{\prime}}}, n_{l}$ and $n_{l^{\prime}}$ being the number of unknowns per box in the respective levels. As every box at level $l$ interacts with at most $C_{\text {int }}$ other boxes at level $l^{\prime}$, the total number of computations between levels $l$ and $l^{\prime}$ scales as $C_{i n t}^{2} \sqrt{n_{l} n_{l^{\prime}}}$. Interactions occur up to level $L-1$. The total number of operations $C C$ is now found by summing over all pairs of levels $l$ and $l^{\prime}$, i.e.,

$$
C C=C_{i n t}^{2} \sum_{l, l^{\prime}=1}^{L-1} \sqrt{n_{l} n_{l^{\prime}}}
$$


The number of unknowns in a box at the lowest level is always fixed, say $m$. This number stems, traditionally, from a $\lambda / 10$ discretization. As in a densely packed quad-tree, the number of unknowns grows by a factor of four at each level $n_{l}=4^{l} m$. Consequently, (25) reduces to

$$
C C=C_{\text {int }}^{2} m\left(2^{L}-2\right)^{2} .
$$

Moreover, at the highest level $L$, all unknowns are members of one big box, thus $4^{L} m=n$. This leads to

$$
C C=C_{i n t}^{2} m\left(\sqrt{\frac{n}{m}}-2\right)^{2}
$$

from which we conclude that $C C \propto \mathcal{O}(n)$.

\section{NUMERICAL RESULTS}

We present three examples. First, the numerical accuracy of our method is assessed by comparing the results of our method to the MoM-UTD scheme in [2] and to an alternative MLFMM-UTD formalism that is somewhat similar to the one presented in [14] as detailed below. Next, the efficiency of our method is demonstrated. Finally, an application example is shown, encompassing two large antenna arrays.

\section{A. Accuracy Test}

In the first example, the NLoS configuration depicted in Fig. 7 is investigated. We explicitly opt for an NLoS configuration because the diffraction contribution is then dominant. Two PEC horn antennas are in NLoS due to the presence of an infinite $90^{\circ}$ PEC wedge. The geometry of the 2-D horns is detailed in Fig. 8. A $2 \lambda$ long parallel-plate, where $\lambda$ is the wavelength in the background medium, serves as a waveguide that suppresses higher order modes and supports the propagation of the fundamental mode. The opening of the horn is tapered. The transmitting antenna is fed by a $1-\mathrm{A} / \mathrm{m}$ line source at a quarter wavelength from its back-end. The center-of-mass (CoM) of the emitter resides at a distance of $\Delta=30 \lambda$ from the tip of the wedge at an angle of $45^{\circ}$ with respect to the upper face of the wedge. The receiving antenna's CoM resides at the same distance $\Delta$ and at an angle of $30^{\circ}$ with respect to the left face of the wedge. Both antennas are directed toward the tip of the wedge. Each antenna is discretized into 105 segments of length $\lambda / 15$. The box size at the lowest level in the MLFMM tree is $\lambda / 2$. The amplitude of the currents along the surface of the antennas, computed by our method, is shown in Fig. 7.

As a reference solution, we implemented the classical MoMUTD [2]. Additionally, for further comparison, we also implemented an alternative 2-D hybrid MLFMM-UTD scheme that is somewhat similar to the one presented in [14]. In this alternative scheme, couplings through reflection and diffraction are treated by traditional UTD from the centers of the interacting boxes. The incoming field at the reflection/diffraction point $\rho$ is determined by employing the following far-field approximation (see also Fig. 2):

$$
G^{f r e e}\left(\boldsymbol{\rho} ; \boldsymbol{\rho}^{\prime}\right) \approx e^{j \boldsymbol{k}_{i} \cdot\left(\boldsymbol{\rho}^{\prime}-\boldsymbol{\rho}_{s}^{c}\right)} T_{L}^{F F}
$$

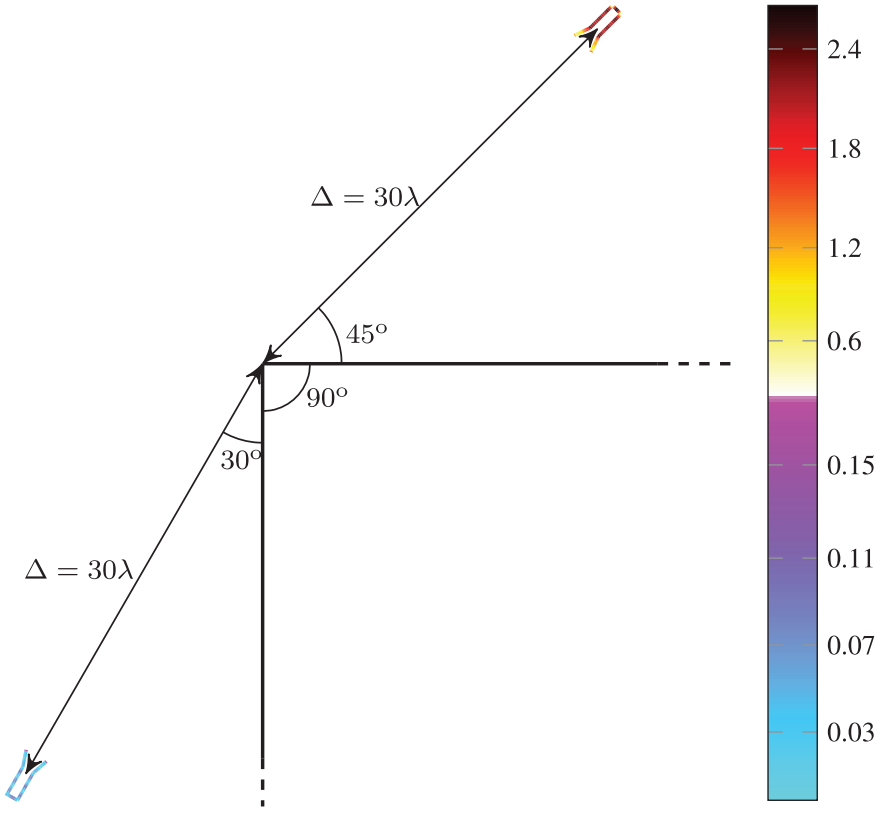

Fig. 7. Amplitude of the unknown current expansion coefficients on the surface of the horns (in $\mathrm{A} / \mathrm{m}$ ). The currents were computed using our MLFMM-UTD method. $\lambda$ is the wavelength in the background medium.

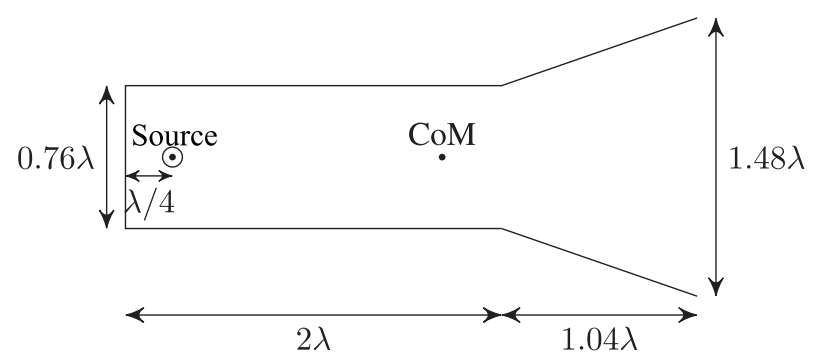

Fig. 8. Geometric details of the horns used in Fig. 7.

where $k_{i}$ is a vector that points from the center of the source box $\rho_{s}^{c}$ to the reflection/diffraction point $\rho$ and $\left|\boldsymbol{k}_{\boldsymbol{i}}\right|=k$. Moreover, $T_{L}^{F F}$ is the 2-D far-field translation operator

$$
T_{L}^{F F}=\frac{1+j}{\sqrt{\pi k}} \frac{e^{-j k r}}{\sqrt{r}}
$$

with $r=\left|\boldsymbol{\rho}-\boldsymbol{\rho}_{\boldsymbol{s}}^{c}\right|$. The required direction of incidence $k_{i}$ is interpolated from the available OPWs by Lagrange interpolation using three interpolation points. After reflection/diffraction, the appropriate ray contribution toward the center of the observation box is projected onto the three neighboring IPWs.

The current density obtained on the 210 segments of the horns was computed with the three techniques. For each segment, the relative error (RE) is defined as

$$
\mathrm{RE}=\left|\frac{J_{z, \text { hybrid }}-J_{z, M o M-U T D}}{J_{z, M o M-U T D}}\right|
$$

where $J_{z, \text { hybrid }}$ is the result from either our newly proposed hybrid technique or from the alternative scheme. $J_{z, M o M-U T D}$ is obtained by means of the reference MoM-UTD [2]. 


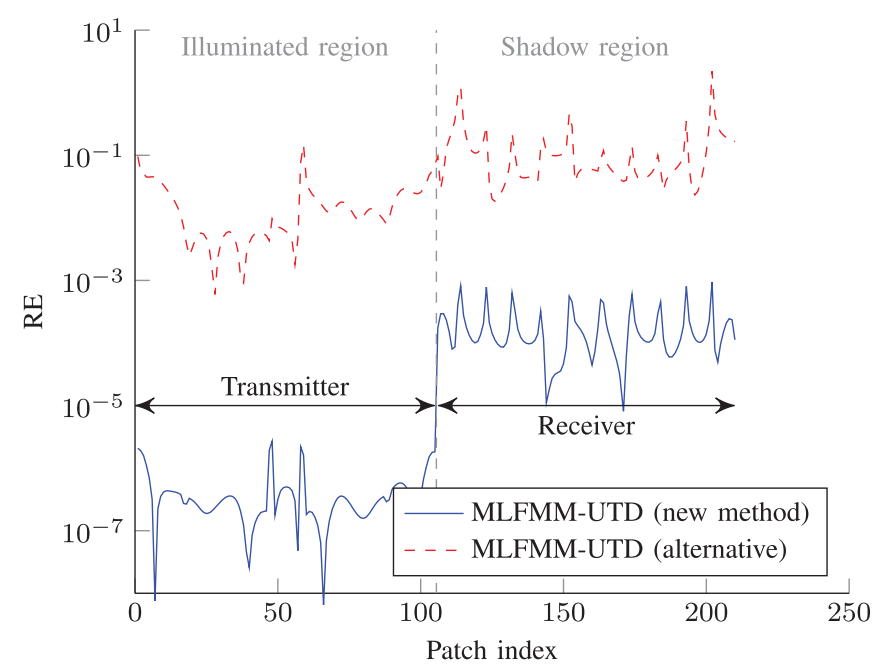

Fig. 9. Relative error on the currents compared to the MoM-UTD solution.

In the illuminated region, in which the transmitter resides, direct and reflection contributions dominate diffraction contributions. As shown in Fig. 9, our method proves to be very accurate in this illuminated region, as reflections are also taken exactly into account. The alternative method uses the canonical UTD solutions to account for reflections. This explains the large difference in accuracy between the two methods for the illuminated part. In the shadow region, where the receiver horn resides, the accuracy is purely determined by the diffraction contribution. It is seen that an RE of less than $0.1 \%$ is easily reached with our new technique. The alternative MLFMMUTD formalism is able to model the amplitude of the field, but fails to accurately predict the phase of the field. This leads to the poorer RE results.

The test is now repeated for a range of values of $\Delta$ and the results are shown in Fig. 10. The mean RE over the patches of each of the antennas is plotted. This mean RE defined as

$$
\text { Mean } \mathrm{RE}=\frac{1}{N} \sum_{i=1}^{N} \mathrm{RE}_{i}
$$

where the summation runs over all patches of the pertinent horn. The accuracy of both MLFMM-UTD schemes increases with increasing distance, as the far-field conditions on which UTD relies are better fulfilled.

\section{B. Efficiency Test}

To test the computational complexity of our method, we consider two $m \times m$ arrays of PEC plates, as shown in Fig. 11 . The length of each plate is $0.5 \lambda$, with $\lambda$ the wavelength in the background medium. The distance between the CoMs of neighboring plates is $0.6 \lambda$. The two arrays are separated by a half infinite, infinitely thin PEC plate. We increase $m$ from 1 to 365 . The size of the largest array is then $218.4 \lambda \times 218.9 \lambda$. The closest point of both arrays to the tip of the half-infinite scatterer is fixed at $10 \lambda$. The box size at the lowest level is $\lambda / 4$. To each plate, five basis functions are assigned. The single process jobs were performed on a processor of an Intel Xeon CPU E5-2680 12-core machine at $2.5 \mathrm{GHz}$ with $512 \mathrm{~GB}$ of RAM.

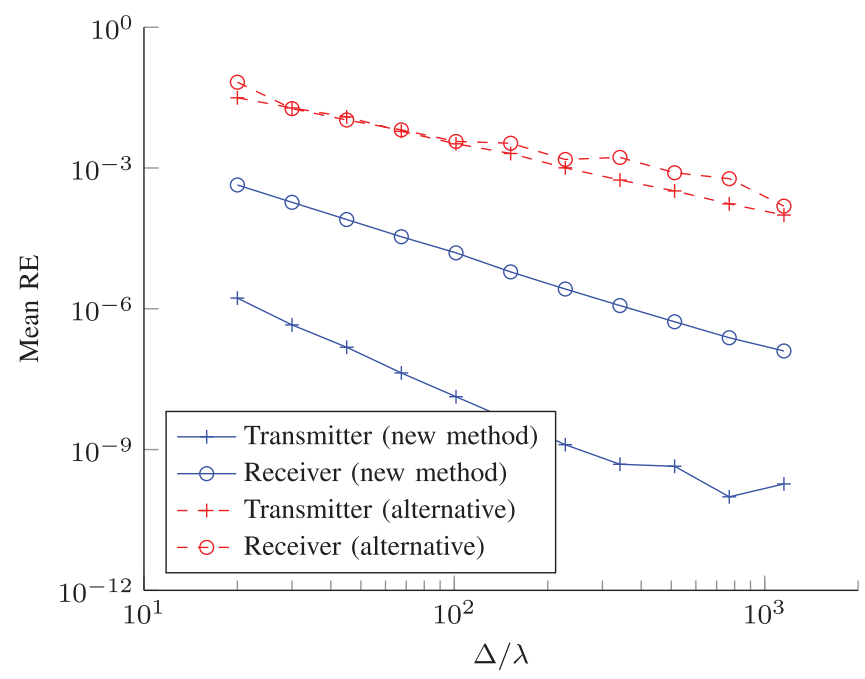

Fig. 10. Mean RE as a function of $\Delta$.

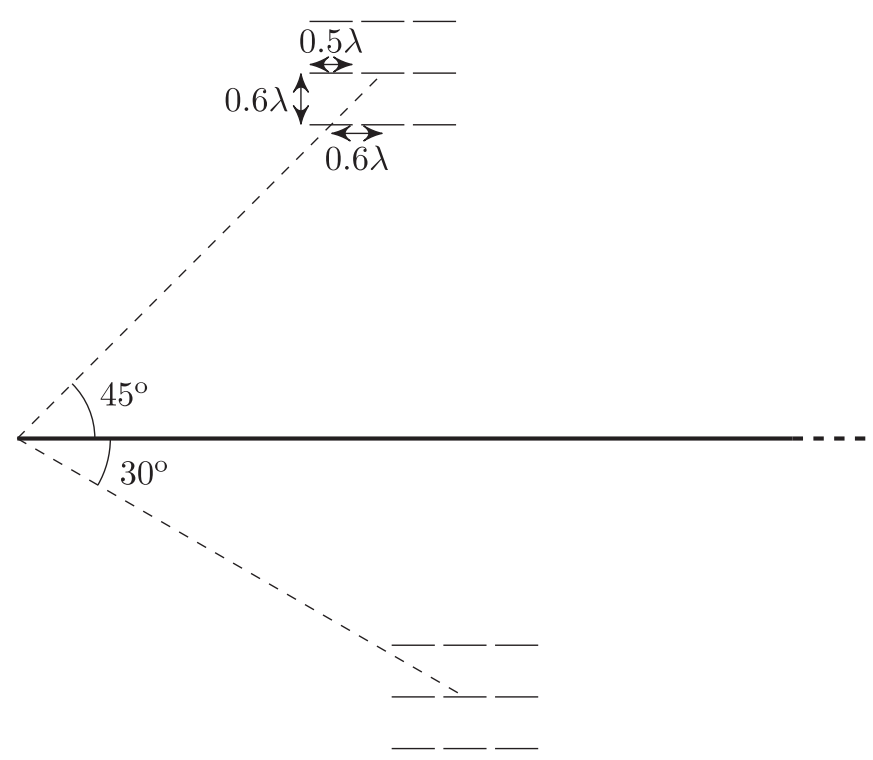

Fig. 11. Geometry for the complexity tests. The plates form two $m \times m$ arrays, where here $m=3$. The arrays are separated by a long straight PEC scatterer.

In Fig. 12, the scaling of the CPU time during the setup phase of the algorithm (i.e., allocating memory, filling up matrices, etc.), the CPU time for one matrix-vector multiplication and the allocated memory are plotted. For our newly proposed MLFMM-UTD method and the alternative MLFMM-UTD method, the CPU time and the memory requirements scale as $\mathcal{O}(n)$. The MoM-UTD scheme [2] has an $\mathcal{O}\left(n^{2}\right)$ complexity. Even for a very low number of unknowns, both MLFMM-UTD schemes are already faster than MoM-UTD during setup. This is due to the dominant contribution of the computation of the UTD coefficients in MoM-UTD. For the matrix-vector multiplication time and the memory requirements, the cross-over point for our novel method is found at about 2500 unknowns. These results are in line with conventional MLFMMs and demonstrate that our MLFMM-UTD method allows the modelling of very large structures. 


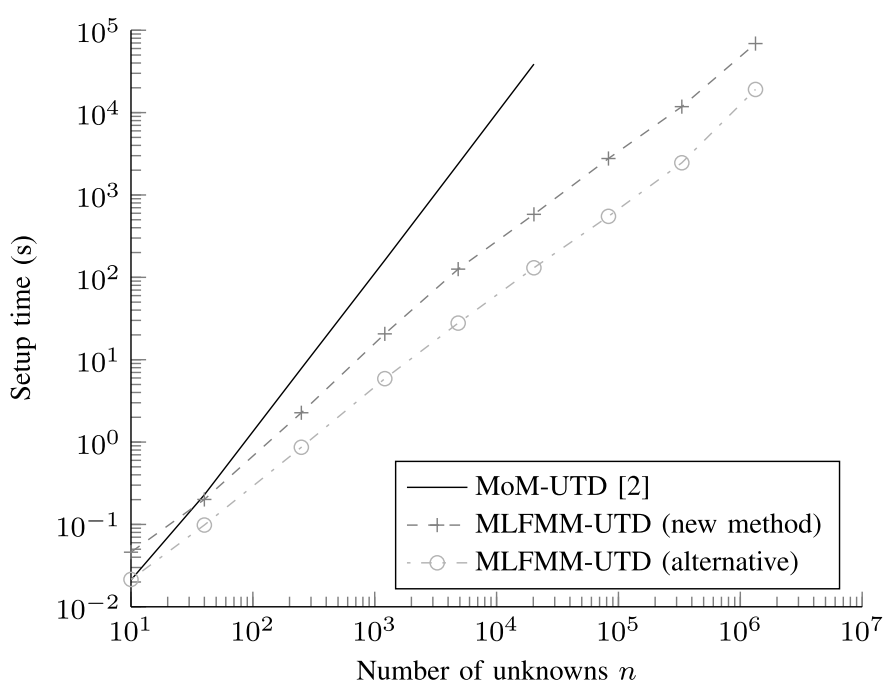

(a)

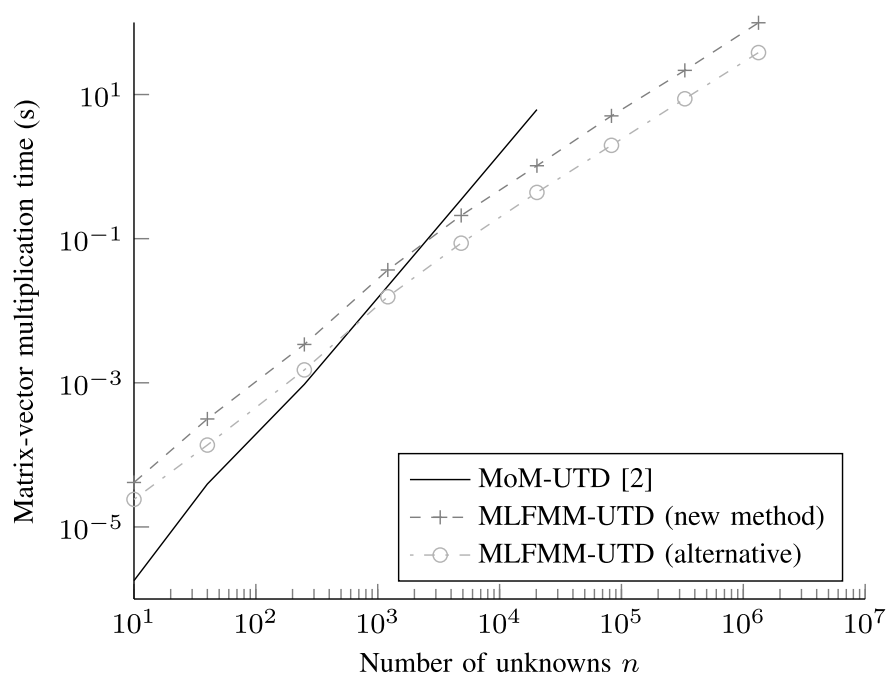

(b)

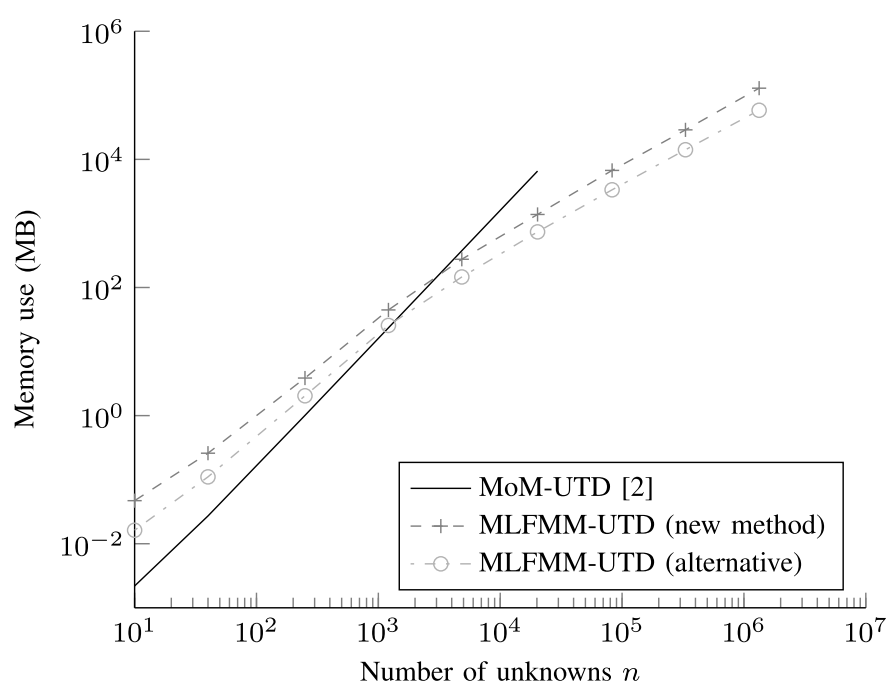

(c)

Fig. 12. Scaling of the resources for an increasing number of unknowns. (a) CPU time during the setup phase. (b) CPU time for one matrix-vector multiplication. (c) Allocated memory.

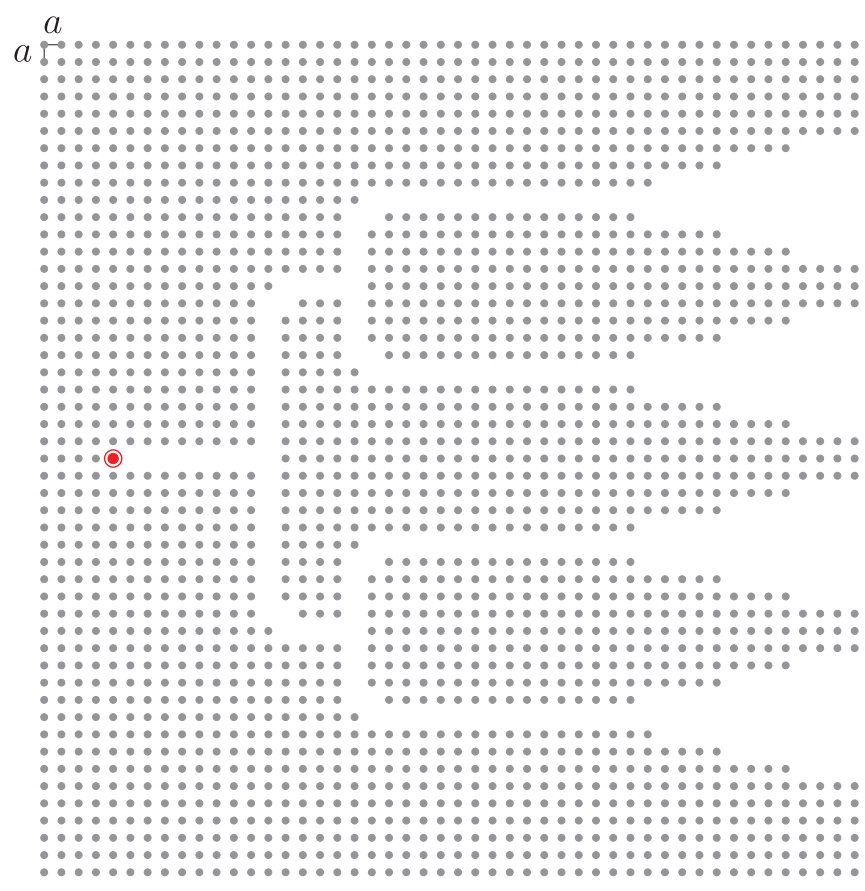

Fig. 13. Horn-antenna array consisting of 1978 PEC cylinders (gray). The array is fed by a line source at the location of the red circle.

\section{Application Example}

In this section, we investigate a structure that illustrates the applicability of the proposed MLFMM-UTD scheme for problems including very large scatterers. The example consists of two radiating horn-antenna arrays that are constructed using a rectangular lattice of PEC circular cylinders. The geometry of one such a horn-antenna array is shown in Fig. 13. By introducing line defects through the lattice, a feed network of waveguides is constructed. The waveguides lead to the front of the lattice, where horns are constructed by gradually tapering the openings. Such structures have been presented in the literature in the context of electromagnetic crystals [19].

The taper length for the horns is $13 a$, with an opening angle of $\arctan (3 / 13) \approx 13^{\circ}$, where $a=0.4 \lambda$ is the distance between the center of two neighbouring cylinders and $\lambda$ is the wavelength in the background medium. The radius of the cylinders is $0.08 \lambda$. We adopt the constellation of Fig. 11 again, but replace the plate arrays by the horn-antenna arrays shown in Fig. 13. Both horn-antenna arrays are oriented toward the tip of the plate. Each horn antenna comprises 1978 cylinders. Ten unknowns are introduced on the surface of each cylinder. In total, the problem consists of $n=39560$ unknowns. The top array is excited by a line source with a current density of $1 \mathrm{~A} / \mathrm{m}$ (see also Fig. 13). Both horn antennas' COMs reside at a distance of $200 \lambda$ from the tip of the wedge. The total computation time was $35 \mathrm{~min}$ and the required memory was about $500 \mathrm{MB}$. The amplitude of the field in the vicinity of the two arrays is shown in Fig. 14. In the shadow, the transmitted field still reaches the receiver via diffraction. The field penetrates the feed network through the separate horns and propagates further into the structure. The contributions of the four horns are 


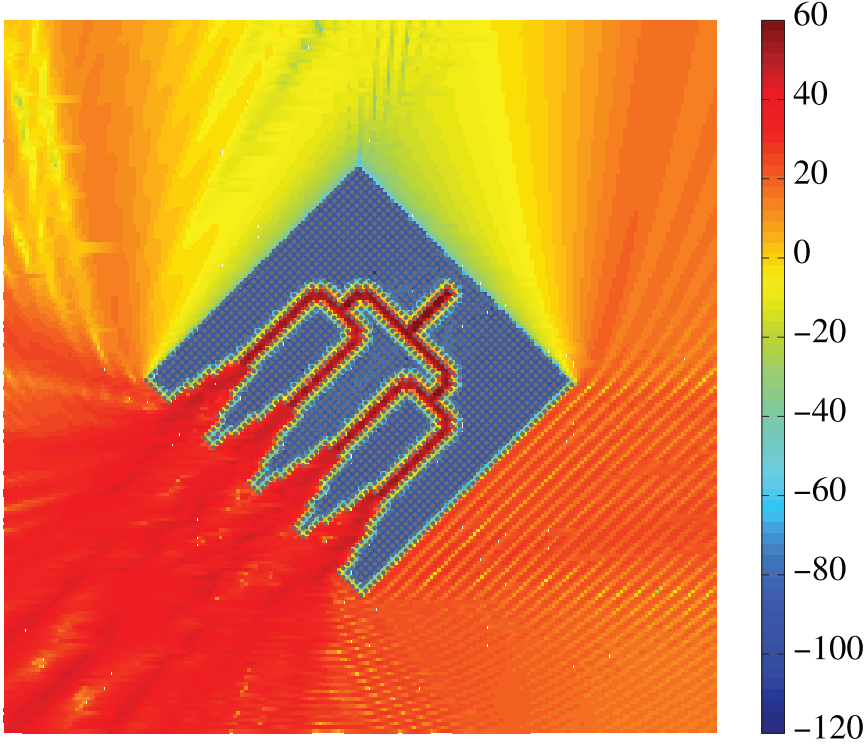

(a)

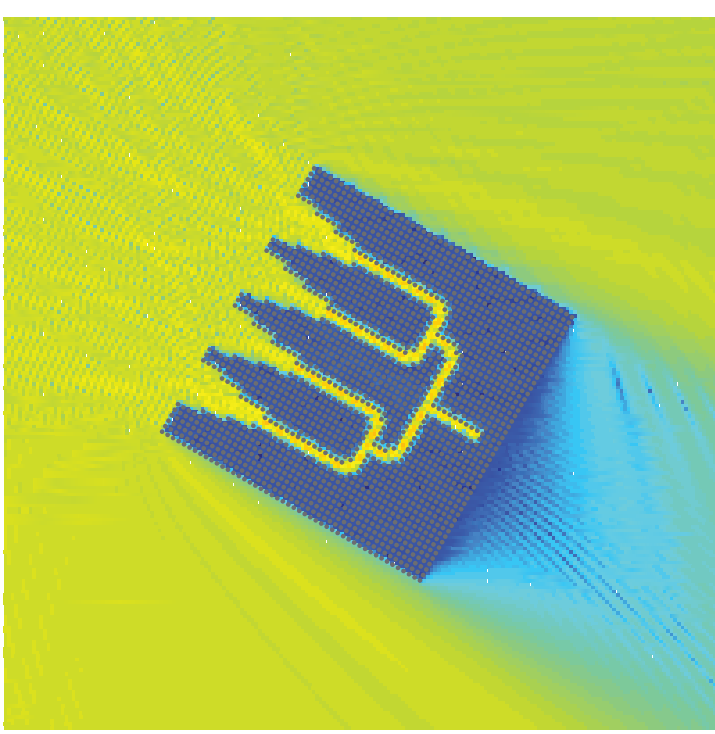

60
40
20
0
-20
-40
-60
-80
-100
-120

(b)

Fig. 14. Amplitude of the electric field (in $\mathrm{dB} \backslash, \mathrm{V} / \mathrm{m}$ ) in the vicinity of the horn antenna arrays. (a) Transmitting array. (b) Receiving array.

clearly combined at the back-end of the network. The reader notices that these fine details can easily be captured by the MLFMM-accelerated BIE-MoM part of our advocated hybrid scheme.

\section{CONCLUSION}

A novel MLFMM-UTD hybrid method has been presented to analyze 2-D scattering in the presence of very large PEC scatterers. Reflections off the surface of the scatterers are taken into account using image theory, as such allowing to maintain the traditional MLFMM accuracy and efficiency via an FFT-based rotation of the pertinent radiation patterns. A new MLFMM-compatible formalism has been introduced to account for diffractions at sharp edges, by generalizing the use of UTD for arbitrary source configurations. The accuracy has been tested and the results correspond well to these of traditional ray-optical hybrid schemes such as MoM-UTD, while the algorithm is much faster and requires a smaller amount of memory. Our novel scheme is accurate for NLoS problems while we also preserve the $\mathcal{O}(n)$ complexity. An application example consisting of electrically large antenna arrays has been simulated to demonstrate the capability of the method. The proposed method is especially suited to model large NLoS scattering problems.

Future work will comprise the extension to 3-D problems. Several issues need to be addressed, such as the rotation of the 3-D radiation pattern when dealing with reflections and the derivation of a new Huyghens' expansion to tackle diffraction. Moreover, a scalable version of the code over multiple processes will be implemented to allow the simulation of larger structures with hundreds of millions and even billions of unknowns [20].

\section{APPENDIX A}

\section{RELATIONSHIP BETWEEN THE COEFFICIENTS $a_{q^{\prime}}$ IN MULTIPOLE EXPANSION (11) AND THE OPWs}

We determine the connection between coefficients $a_{q^{\prime}}$ in (11) and the OPWs in the far-field. The asymptotic approximation for the cylindrical harmonics $H_{q^{\prime}}^{(2)}\left(k\left\|\rho_{o}-\rho_{s}^{c}\right\|\right)$ in (11), for large argument values, is [18]

$$
H_{\nu}^{(2)}(z) \approx \frac{1+j}{\sqrt{\pi z}} e^{-j z} e^{j \nu \pi / 2},|z| \gg \nu .
$$

Inserting (A1) into (11) results in

$$
E^{i n c}\left(\boldsymbol{\rho}_{\boldsymbol{o}}\right) \approx-\frac{1+j}{\sqrt{\pi k}} \frac{\omega \mu_{0}}{4} \frac{e^{-j k\left\|\boldsymbol{\rho}_{o}-\boldsymbol{\rho}_{s}^{\boldsymbol{c}}\right\|}}{\sqrt{\left\|\boldsymbol{\rho}_{\boldsymbol{o}}-\boldsymbol{\rho}_{\boldsymbol{s}}^{\boldsymbol{c}}\right\|}} \sum_{q^{\prime}=-Q^{\prime}}^{Q^{\prime}} a_{q^{\prime}} e^{j q^{\prime}\left(\phi_{o}+\pi / 2\right)} .
$$

In the presence of a single line source with current density $J_{s}$ residing at $\rho^{\prime}\left(\left\|\rho^{\prime}-\rho_{s}^{c}\right\|<\left\|\rho_{o}-\rho_{s}^{c}\right\|\right), E^{i n c}\left(\rho_{o}\right)$ is also given by

$$
E^{i n c}\left(\boldsymbol{\rho}_{\boldsymbol{o}}\right)=-J_{s} \frac{\omega \mu_{0}}{4} H_{0}^{(2)}\left(k\left\|\boldsymbol{\rho}_{\boldsymbol{o}}-\boldsymbol{\rho}^{\prime}\right\|\right) .
$$

In the far-field, using (A1), the asymptotic approximation becomes

$$
E^{i n c}\left(\boldsymbol{\rho}_{\boldsymbol{o}}\right) \approx-J_{s} \frac{1+j}{\sqrt{\pi k}} \frac{\omega \mu_{0}}{4} \frac{e^{-j k\left\|\boldsymbol{\rho}_{o}-\boldsymbol{\rho}^{\prime}\right\|}}{\sqrt{\left\|\boldsymbol{\rho}_{o}-\boldsymbol{\rho}^{\prime}\right\|}} .
$$

We further introduce the Taylor expansion

$$
\begin{array}{r}
\left\|\rho_{o}-\rho^{\prime}\right\|>=\left\|\rho_{o}-\rho_{s}^{c}-\left(\rho^{\prime}-\rho_{s}^{c}\right)\right\| \\
\approx\left\|\rho_{o}-\rho_{s}^{c}\right\|-\hat{k}\left(\phi_{o}\right) \cdot\left(\rho^{\prime}-\rho_{s}^{c}\right) \\
+\mathcal{O}\left(\left\|\rho_{o}-\rho_{s}^{c}\right\|^{-1}\right)
\end{array}
$$

in (A4), with $\hat{\boldsymbol{k}}\left(\phi_{o}\right)=\cos \phi_{o} \hat{\boldsymbol{x}}+\sin \phi_{o} \hat{\boldsymbol{y}}$. For the amplitude, we use only the zeroth-order approximation, while for the phase we keep the first-order term as well yielding

$$
E^{i n c}\left(\boldsymbol{\rho}_{\boldsymbol{o}}\right) \approx-\frac{1+j}{\sqrt{\pi k}} \frac{\omega \mu_{0}}{4} \frac{e^{-j k\left\|\boldsymbol{\rho}_{o}-\boldsymbol{\rho}_{s}^{c}\right\|}}{\sqrt{\left\|\boldsymbol{\rho}_{o}-\boldsymbol{\rho}_{\boldsymbol{s}}^{c}\right\|}} \mathcal{F}\left(\phi_{o}\right)
$$


in which $\mathcal{F}\left(\phi_{o}\right)=J_{s} e^{j \boldsymbol{k}\left(\phi_{o}\right) \cdot\left(\boldsymbol{\rho}^{\prime}-\boldsymbol{\rho}_{s}^{c}\right)}$ and $\boldsymbol{k}\left(\phi_{o}\right)=k \hat{\boldsymbol{k}}\left(\phi_{o}\right)$. In the case of multiple sources, (A6) remains valid, provided that $\mathcal{F}\left(\phi_{o}\right)$ is replaced by a superposition of factors similar to the one derived here. The function $\mathcal{F}\left(\phi_{0}\right)$ describes the radiation pattern of box $B^{\prime}$. Comparison with (5) shows that the OPWs are sampled values of this radiation pattern. As $\mathcal{F}\left(\phi_{o}\right)$ is quasi-bandlimited, it can be reconstructed from the OPWs [21]. Thereto, the appropriate kernel is the Dirichlet kernel, given by

$$
D(\phi)=\sum_{q^{\prime}=-Q^{\prime}}^{Q^{\prime}} \frac{e^{j q^{\prime} \phi}}{2 Q^{\prime}+1} .
$$

The reconstruction is performed in the following way:

$$
\begin{aligned}
\mathcal{F}\left(\phi_{o}\right) \approx & \sum_{p^{\prime}=-Q^{\prime}}^{Q^{\prime}} \mathrm{OPW}_{p^{\prime}} D\left(\phi_{o}-\phi_{p^{\prime}}\right)=\frac{1}{2 Q^{\prime}+1} \sum_{q^{\prime}=-Q^{\prime}}^{Q^{\prime}} e^{j q^{\prime} \phi_{o}} \\
& \times \sum_{p^{\prime}=-Q^{\prime}}^{Q^{\prime}} \mathrm{OPW}_{p^{\prime}} e^{-j q^{\prime} \phi_{p^{\prime}}} .
\end{aligned}
$$

Introduction of (A8) into (A6) and identification with (A2) finally leads to

$$
a_{q^{\prime}}=\frac{1}{2 Q^{\prime}+1} \sum_{p^{\prime}=-Q^{\prime}}^{Q^{\prime}} \mathrm{OPW}_{p^{\prime}} e^{-j q^{\prime}\left(\phi_{p^{\prime}}+\pi / 2\right)} \text {. }
$$

\section{APPENDIX B \\ Fourier InTEGRAL OF BESSEL FunCtions}

The Bessel functions $J_{q}\left(k\left\|\rho-\rho_{\boldsymbol{o}}^{\boldsymbol{c}}\right\|\right)$ in (12) can be described by a Fourier integral [18]

$J_{q}\left(k\left\|\boldsymbol{\rho}-\boldsymbol{\rho}_{\boldsymbol{o}}^{\boldsymbol{c}}\right\|\right)=\frac{1}{2 \pi} \int_{2 \pi} \mathrm{d} \phi^{*} e^{-j \boldsymbol{k}\left(\phi^{*}\right) \cdot\left(\boldsymbol{\rho}-\boldsymbol{\rho}_{\mathrm{o}}^{\boldsymbol{c}}\right)} e^{j q\left(\phi^{*}-\phi+\pi / 2\right)}$.

The integral can also be discretized to yield a summation [22], yielding

$$
\begin{aligned}
& J_{q}\left(k\left\|\boldsymbol{\rho}-\boldsymbol{\rho}_{\boldsymbol{o}}^{\boldsymbol{c}}\right\|\right) e^{j q \phi}=\frac{1}{2 Q+1} \\
& \times \sum_{p=-Q}^{Q} e^{-j \boldsymbol{k}\left(\phi_{p}\right) \cdot\left(\boldsymbol{\rho}-\boldsymbol{\rho}_{\boldsymbol{o}}^{\boldsymbol{c}}\right)} e^{j q\left(\phi_{p}+\pi / 2\right)}
\end{aligned}
$$

\section{ACKNOWLEDGMENT}

The computational resources (STEVIN Supercomputer Infrastructure) and services used in this work were kindly provided by Ghent University, the Flemish Supercomputer Center (VSC), the Hercules Foundation and the Flemish Government-department EWI.

\section{REFERENCES}

[1] W. C. Chew, J.-M. Jin, E. Michielssen, and J. Song, Fast and Efficient Algorithms in Computational Electromagnetics. Norwood, MA, USA: Artech House, 2001.
[2] G. A. Thiele and T. H. Newhouse, "A hybrid technique for combining moment methods with the geometrical theory of diffraction," IEEE Trans. Antennas Propag., vol. 23, no. 1, pp. 62-69, Jan. 1975.

[3] O. A. Civi, P. H. Pathak, H. T. Chou, and P. Nepa, "A hybrid uniform geometrical theory of diffraction-moment method for efficient analysis of electromagnetic radiation/scattering from large finite planar arrays," Radio Sci., vol. 35, no. 2, pp. 607-620, 2000.

[4] H. F. Harms, "Using wire grid structures in the combination of uniform geometric theory of diffraction and method of moments for near-field analysis," Radio Sci., vol. 35, no. 2, pp. 639-652, 2000.

[5] C. J. Reddy, M. D. Deshpande, C. R. Cockrell, and F. B. Beck, "Radiation characteristics of cavity backed aperture antennas in finite ground plane using the hybrid FEM/MOM technique and geometrical theory of diffraction," IEEE Trans. Antennas Propag., vol. 44, no. 10, pp. 1327-1333, Oct. 1996.

[6] J. Silvestro, "Scattering from slot near conducting wedge using hybrid method of moments geometrical-theory of diffraction - TE case," Electron. Lett., vol. 28, no. 11, pp. 1055-1057, 1992.

[7] I. P. Theron, D. B. Davidson, and U. Jakobus, "Extensions to the hybrid method of moments/uniform GTD formulation for sources located close to a smooth convex surface," IEEE Trans. Antennas Propag., vol. 48, no. 6, pp. 940-945, Jun. 2000.

[8] X. C. Wei and E. P. Li, "Efficient EMC simulation of enclosures with apertures residing in an electrically large platform using the MM-UTD method," IEEE Trans. Electromagn. Compat., vol. 47, no. 4, pp. 717-722, Nov. 2005.

[9] W. D. Burnside, C. L. Yu, and R. J. Marhefka, "Technique to combine the geometrical theory of diffraction and moment method," IEEE Trans. Antennas Propag., vol. 23, no. 4, pp. 551-558, Jul. 1975.

[10] H. J. Bilow, "Scattering by an infinite wedge with tensor impedance boundary conditions - A moment method/physical optics solution for the currents," IEEE Trans. Antennas Propag., vol. 39, no. 6, pp. 767-773, Jun. 1991.

[11] Z. Q. Gong, B. X. Xiao, G. Q. Zhu, and H. Y. Ke, "Improvements to the hybrid MoM-PO technique for scattering of plane wave by an infinite wedge," IEEE Trans. Antennas Propag., vol. 54, no. 1, pp. 251-255, Jan. 2006.

[12] E. Jørgensen, S. Maci, and A. Toccafondi, "Fringe integral equation method for a truncated grounded dielectric slab," IEEE Trans. Antennas Propag., vol. 49, no. 8, pp. 1210-1217, Aug. 2001.

[13] G. Apaydin, F. Hacivelioglu, L. Sevgi, and P. Ufimtsev, "Wedge diffracted waves excited by a line source: Method of moments (MOM) modeling of fringe waves," IEEE Trans. Antennas Propag., vol. 62, no. 8, pp. 43684371, Aug. 2014.

[14] A. Tzoulis and T. F. Eibert, "A hybrid FEBI-MLFMM-UTD method for numerical solutions of electromagnetic problems including arbitrarily shaped and electrically large objects," IEEE Trans. Antennas Propag., vol. 53, no. 10, pp. 3358-3366, Oct. 2005.

[15] G. Karagounis, D. De Zutter, and D. Vande Ginste, "Computation of the diffraction from complex illumination sources in extended regions of space," Opt. Express, vol. 21, no. 25, pp. 30 379-30 391, 2013.

[16] D. Vande Ginste, E. Michielssen, F. Olyslager, and D. De Zutter, "A high-performance upgrade of the perfectly matched layer multilevel fast multipole algorithm for large planar microwave structures," IEEE Trans. Antennas Propag., vol. 57, no. 6, pp. 1728-1739, Jun. 2009.

[17] I. Bogaert, D. Pissoort, and F. Olyslager, "A normalized plane-wave method for 2D helmholtz problems," Microw. Opt. Technol. Lett., vol. 48, no. 2 , pp. 237-243, 2006.

[18] "NIST digital library of mathematical functions," Release 1.0.5, Oct. 1, 2012, Online companion to [23] [Online]. Available: http://dlmf.nist.gov/

[19] D. Pissoort, E. Michielssen, D. Vande Ginste, and F. Olyslager, "A rank-revealing preconditioner for the fast integral-equation-based characterization of electromagnetic crystal devices," Microw. Opt. Technol. Lett., vol. 48, pp. 783-789, 2006.

[20] B. Michiels, J. Fostier, I. Bogaert, and D. De Zutter, "Full-wave simulations of electromagnetic scattering problems with billions of unknowns," IEEE Trans. Antennas Propag., vol. 63, no. 2, pp. 796-799, Feb. 2015.

[21] D. Vande Ginste, L. Knockaert, and D. De Zutter, "Error control in the perfectly matched layer based multilevel fast multipole algorithm," $J$. Comput. Phys., vol. 228, no. 13, pp. 4811-4822, 2009.

[22] R. J. Burkholder and D. H. Kwon, "High-frequency asymptotic acceleration of the fast multipole method," Radio Sci., vol. 31, no. 5, pp. 1199-1206, 1996.

[23] F. W. J. Olver, D. W. Lozier, R. F. Boisvert and C. W. Clark, Eds., NIST Handbook of Mathematical Functions. Cambridge, U.K.: Cambridge Univ. Press, 2010, print companion to [18]. 


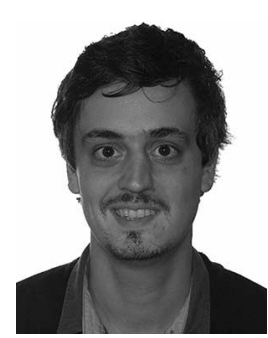

Giorgos Karagounis was born in 1988 in Athens, Greece. He received the M.Sc. degree in applied physics from the University of Gent, Ghent, Belgium, in 2012. Since October 2012, he has been pursuing the $\mathrm{Ph} . \mathrm{D}$. degree at the Electromagnetics Group, Department of Information Technology, University of Gent.

His research interests include computational electromagnetics and more specifically hybrid highfrequency methods.

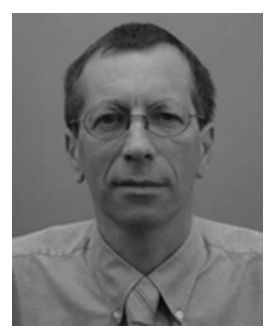

Daniël De Zutter (F'00) was born in 1953. He received the M.Sc. degree in electrical engineering, the $\mathrm{Ph} . \mathrm{D}$. degree, and the degree (equivalent to the French Aggregation or German Habilitation) from the University of Gent, Ghent, Belgium, in 1976, 1981, and 1984, respectively.

He served as the Dean of the Faculty of Engineering with Ghent University from 2004 to 2008, where he is currently the Head of the Department of Information Technology. He is also a Full Professor of Electromagnetics. He has authored or coauthored over 200 international journal papers (cited in the Web of Science), and 200 papers in conference proceedings. His research interests include circuit and electromagnetic modeling of high-speed and high-frequency interconnections and packaging, on electromagnetic compatibility and numerical solutions of Maxwells equations.

Dr. Zutter was an Associate Editor of the IEEE TRANSACTIONS ON MicrowaVe THEORY AND TECHNIQUeS.

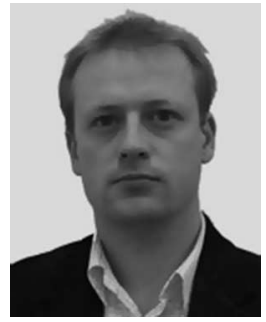

Dries Vande Ginste (SM'12) received the M.S. and $\mathrm{Ph} . \mathrm{D}$. degrees in electrical engineering from Ghent University, Gent, Belgium, in 2000 and 2005, respectively.

$\mathrm{He}$ is currently an Associate Professor with the Department of Information Technology, Ghent University and a Guest Professor at iMinds. In June and July 2004, he was a Visiting Scientist at the Department of Electrical and Computer Engineering, University of Illinois at Urbana-Champaign (UIUC), IL, USA. From September to November 2011, he was a Visiting Professor at the EMC Group, Dipartimento di Elettronica, Politecnico di Torino, Torino, Italy. He has authored or coauthored over 100 papers in international journals and in conference proceedings. His research interests include computational electromagnetics, electromagnetic compatibility, signal and power integrity, and antenna design.

Dr. Vande Ginste served as the Co-Chair of the 2014 IEEE Workshop on Signal and Power Integrity. He was the recipient of the International Union of Radio Science (URSI) Young Scientist Award at the 2011 URSI General Assembly and Scientific Symposium, the Best Poster Paper Award at the 2012 IEEE Electrical Design of Advanced Packaging and Systems Symposium (EDAPS), the Best Paper Award at the 2013 IEEE Workshop on Signal and Power Integrity (SPI) and the Best Paper Award at the 2013 IEEE International Conference on Electrical Performance of Electronic Packaging and Systems (EPEPS). 